Rev.MVZ Córdoba 18(1):3257-3258, 2013.

EDITORIAL

\title{
Universidades colombianas. Ranking Iberoamericano SIR 2012. ¿Para donde vamos?
}

\section{Colombian universities. Latin American Ranking SIR 2012. Where are we going?}

La calidad de la educación indudablemente tiene que ser evaluada y medida. Sin embargo, al ser objeto de medición se aplican algunos criterios bibliométricos medibles que pretenden ser objetivos pero algunas veces no son del agrado en algunos sectores académicos. El fenómeno de la calidad de la educación superior es complejo y está influenciado por las diferencias sociales y económicas globales, así como también por las condiciones individuales de trabajo y presupuestales de cada universidad en particular $(1,2)$.

Las clasificaciones o rankings son criticadas por las metodologías utilizadas y también por las diferencias existentes entre las universidades del mundo. Sin embargo, aunque haya disensión entre la comunidad académica (administradores, investigadores, estudiantes), éstas clasificaciones, finalmente, son interesantes y se utilizan como una herramienta que permite orientar las estrategias a fin de aumentar la visibilidad y competitividad de las universidades (3). Las universidades, por pequeñas que sean, no pueden ignorar esta realidad en un mundo cada vez más globalizado, y por tanto, están llamadas en su conjunto a diseñar estrategias administrativas y académicas que apunten hacia la consecución de los criterios que tienen en cuenta algunos de los rankings de acuerdo con las posibilidades de acceso de cada universidad. Naturalmente, los planes y estrategias deberán estar articulados con los de Ciencia y Tecnología promulgados y financiados por los organismos responsables de este sector.

Para el caso del ranking considerado como el más riguroso, el Academic Ranking of World Universities que utiliza criterios tan exigentes como: número de profesores y alumnos que hayan ganado premios Nobel y otros galardones $(10 \%)$, premios Nobel dentro de la planta docente $(20 \%)$, menciones a sus investigadores $(20 \%)$, artículos publicados en revistas científicas de alto factor de impacto $(20 \%)$, documentos indexados en las mejores bases de datos (20\%) y sus resultados per cápita respecto al tamaño de la institución (10\%), las posibilidades para nuestras universidades colombianas se tornan un tanto complicadas.

De todas maneras, para la realidad colombiana, sea cual fuere, se debe reconocer que finalmente estos rankings ofrecen una clara visión de la educación superior en un país y su lugar en el mundo académico.

El Ranking Iberoamericano SCImago (SIR, por su sigla en inglés SCImago Institutions Rankings) (4) es una plataforma que se utiliza como herramienta para analizar la actividad investigadora de las Instituciones de Educación Superior en Iberoamérica. Las clasificaciones incluyen indicadores bibliométricos con el objeto de revelar la magnitud investigadora de las instituciones. Los indicadores utilizados por este ranking para cada institución son cinco. 1. Producción Científica. Número de publicaciones en revistas científicas. 2. Colaboración Internacional. Ratio de publicaciones científicas de una institución elaboradas conjuntamente con instituciones de otros países. 3. Calidad Científica Promedio. Impacto científico de una institución después de eliminar la influencia del tamaño y el perfil temático de la institución. Este indicador permite comparar instituciones de diferentes tamaños y con distintos perfiles de investigación. 4. Porcentaje de Publicaciones en Revistas del Primer Cuartil SJR. Indica el porcentaje de publicaciones que una institución ha publicado en revistas incluidas en el primer cuartil ordenadas por el indicador SJR. (5). 5. Ratio de Excelencia. Indica el porcentaje del output de una institución que se encuentra incluido en el $10 \%$ de los trabajos más citados del mundo en sus respectivos campos científicos. Es un indicador de la producción científica de alta calidad que produce una institución. 
El análisis de los resultados permitirá a los responsables de cada institución (administradores, investigadores, comunicadores de la web institucional) tomar decisiones encaminadas a mejorar o a reorientar la investigación lo que finalmente incrementaría la posibilidad de ascender en la clasificación y en últimas podría mejorar las fuentes de financiación para programas de investigación y extensión.

Recientemente, el Ranking Iberoamericano SCImago publicó la medición de 2012 (4). No sorprende que en esta última clasificación en Colombia, los primeros cinco lugares continúen ocupados por las mismas universidades que habitualmente aparecen: Nacional, Antioquia, Andes, Valle y Javeriana. Posteriormente se encuentra la UIS, Rosario, UPB, Norte y Cauca. En el Caribe, la universidad del Norte ocupó el puesto 9, Cartagena el 13 y Córdoba el 17; estas IES mostraron un avance importante pero aun lejos del top de los cinco primeras. En Iberoamérica tampoco hay mayores cambios, la universidad de Sao Paulo (Brasil), la UNAM (México) y la de Campinas (Brasil) superaron a las españolas de Barcelona y Autónoma de Barcelona y Madrid. Estas universidades tienen más estudiantes de postgrado (M.Sc. y Ph.D.) que generan más conocimiento y por lo tanto, la publicación de mayor número artículos, lo que conlleva a incrementar el prestigio de las mismas en el mundo académico.

Lo importante de los rankings no es el lugar que las universidades alcancen cada año, lo es en realidad el análisis de los indicadores obtenidos por cada institución, lo cual le permitirá trazar políticas y estrategias para mejorarlos en un futuro a corto o mediano plazo. Teniendo en cuenta los indicadores utilizados por la mayoría de los rankings, básicamente, las universidades colombianas deben apostarle principalmente a: i. Contratar o vincular profesores de tiempo completo con posgrado (M.Sc. y Ph.D.) para realizar investigación. ii. Publicar en inglés en revistas indexadas con alto factor de impacto iii. Buscar alianzas estratégicas internacionales para realizar investigación y publicación de artículos en forma conjunta. iv. Desarrollar una decidida y ambiciosa política de publicación de toda la producción científica (Tesis, proyectos de investigación, libros, textos universitarios, monografías, ensayos, guías, etc.) que se desarrolla al interior de la universidad. v. Fortalecer y modernizar las bibliotecas, incluyendo el desarrollo de las virtuales. vi. Desarrollar una WEB moderna, amigable y fundamentalmente bilingüe para que la comunidad internacional tenga acceso a dicha información. No obstante, no se podrán realizar publicaciones de impacto con investigadores internacionales, sin profesores universitarios altamente calificados.

En ese sentido, con solo un $37 \%$ de profesores de planta, será muy difícil que la Universidad de Córdoba supere el puesto 341 en Iberoamérica y el 255 en Latinoamérica. Creemos que la cultura de la mampostería y del ciclópeo debería estar limitada a lo imprescindible y más bien apostarle, al menos por un tiempo estratégico, a construir, si a construir, un pensamiento complejo de universidad que incluya la creación de nuevos posgrados en distintas áreas del conocimiento y desde esta plataforma promover una universidad con mayor liderazgo regional, nacional e internacional.

Salim Mattar V. Ph.D. Marco González T. M.Sc.

\section{REFERENCIAS}

1. Vistremundo Águila C. El concepto calidad en la educación universitaria: clave para el logro de la competitividad institucional. [en línea]. Revista Iberoamericana de Educación, OEI. 2005. [fecha de acceso enero de 2013]. URL Disponible en: http://www.rieoei. org/deloslectores/880Aguila.PDF

2. Ximena Cardoso A. Hacia la internacionalización de la educación superior. [en línea]. Colombia. Ministerio de educación. [fecha de acceso enero de 2013]. URL Disponible en: http://www.mineducacion.gov.co/1621/ articles-230245_archivo_pdf_articulo_ internacionalizacion.pdf
3. Docampo D. "Rankings internacionales y calidad institucional". Revista de Educación 2008; Número Extraordinario:149-176. URL Disponible http://www.revistaeducacion. mec.es/re2008/re2008_07.pdf

4. SCImago Institutions Rankings (SIR). [en línea]. España: SCImago Research Group; 2012. [fecha de acceso enero de 2013].URL Disponible en: http://www.scimagoir.com/ pdf/sir_2012_world_report.pdf

5. SJC. SCImago Journal \& Country Rank. [en línea]. España: SCImago Lab; 2013. [fecha de acceso enero de 2013]. URL Disponible en: http://www.scimagojr.com/ 